Please cite this paper as follows:

Han-Chin Wu, Hong-Ki Hong and Jun-Kai Lu, An Endochronic Theory Accounted for Deformation Induced Anisotropy, International Journal of Plasticity, Vol.11, No.2, pp.145-162, 1995. 


\title{
AN ENDOCHRONIC THEORY ACCOUNTED FOR DEFORMATION INDUCED ANISOTROPY
}

\author{
Han-Chin Wu, ${ }^{*}$ Hong-Ki Hong, and Jun-KaI LU* \\ National Taiwan University
}

\begin{abstract}
A.bstract - An anisotropic quadratic form of plastic strain increment is used to define the intrinsic time in the endochronic theory of plasticity. Based on this new definition, a yield function can be derived. This new version of endochronic theory can describe the expansion, translation, rotation, and distortion of the yield surface. While the initial yielding is in the form of the Mises yield criterion, the distortion of subsequent yield surfaces is expressed by the compression or stretching of the Mises yield surface. The effect of sharp front and blunt rear of the yield surface is considered to be of secondary importance and neglected in the interest of keeping the equations simple. This idealization will not much affect the prediction power of the model, because the plastic strain increment is in the radial direction emanating from the center of the current yield surface and is not normal to the current yield surface. In this theory, the plastic deformation is thus not sensitive to the exact shape of the yield surface. It has been shown that the proposed theory is capable of describing the experimental results of three different metals considered. The test series investigated include several different paths of prestress.
\end{abstract}

\section{INTRODUCTION}

The purpose of this article is to discuss the deformation induced anisotropic behavior of metals in the small strain range by use of the endochronic theory of plasticity. For an initially isotropic material, the deformation induced anisotropy is explicitly shown through the motion and distortion of the yield surface and the flow rule. It has been previously shown by VALANIS [1980] and further discussed by WU and YEH [1987] that yield surface may be deduced from the endochronic constitutive equations. However, in most previous works (VAlanis [1980], Wu et al. [1986], Wu \& YEH [1989]), the hardening rule for the yield surface used combined isotropic-kinematic hardening. The distortion of the yield surface was not accounted for.

WU and YEH [1987] considered the distortion of yield surface by use of a distortion function that is based on the geometric concept of PHILLIPS and WENG [1975] and EISENBERG and YEN [1984]. According to this concept, the distortion function takes different values along different radial lines emanating from the center of the yield surface. WU and YEH [1987] further modified the endochronic equation to account for the strain path dependent rate of kinematic hardening. Therefore, the rate of change of the back stress is anisotropic in this theory. In their formulation, the plastic strain increment, although normal to the initial yield surface, is not normal to subsequent yield surfaces.

In this work, the endochronic constitutive equations are those derived by WU and YEH 
[1987] and account for the anisotropic rate of back stress evolution. However, the isotropic hardening function describes only the effect of isotropic hardening and is not used as in WU and YEH [1987] to describe the distortion of yield surface. Instead, the distortion of yield surface is accounted for by redefining the intrinsic time. It has thus been shown that the form used to define the intrinsic time is closely related to the shape of the yield surface.

The shape of yield surface is known to depend on the prestress history. Even though the Mises yield criterion is generally accepted as providing a reasonable representation of the initial yield surface, it does not describe the subsequent yield surface well. Experimental findings show that the subsequent yield surface can expand in size, translate, distort, and rotate depending on the material being tested and the loading path in the stress space. A brief summary of experimental investigation of yield surface may be found in WU and YEH [1991].

Numerous efforts are found in the literature with the goal of more closely representing the subsequent yield surfaces. These efforts are usually carried out in connection with the classical plasticity theory, which defines a yield surface and a flow rule. The majority of the works have concentrated in the description of the shape and the position of the yield surface and use the usual expression for the flow rule, assuming normality condition for the plastic strain increment with respect to the current yield surface. Some of the researchers proposed yield functions that contain high order terms (the algebraic approach), and others fit the shape and position of subsequent yield surfaces from the geometric viewpoint (the geometric approach). Although the goal of describing the distortion of yield surface has been achieved by some of the models, the complex expressions of the models reduce their usefulness.

In the algebraic approach, BALTov and SAWCZUK [1965] defined a yield function given by

$$
2 F=N_{i j k l}\left(s_{i j}-\alpha_{i j}\right) \cdot\left(s_{k l}-\alpha_{k l}\right)-2 k^{2}=0,
$$

where $N_{i j k l}$ is a fourth order tensor; $s_{i j}$ is the deviatoric stress; $\alpha_{i j}$ is the back stress; $k$ is a scalar function, and $N_{i j k l}$ and $\alpha_{i j}$ are tensorial functions of the plastic strain $\varepsilon_{i j}^{p}$. In addition, $N_{i j k l}$ can be separated into an isotropic part $I_{i j k l}$ and an anisotropic part $A_{i j k l}$. By using the polynomial form and neglecting higher order terms, the anisotropic part is written as $A_{i j k l}=A \varepsilon_{i j}^{p} \varepsilon_{k l}^{p}$, which, when combined with Prager's linear kinematic hardening rule, would make eqn (1) read

$$
2 F=s_{i j} s_{i j}-2 s_{i j} \alpha_{i j}+A \alpha_{i j} \alpha_{k l}\left(s_{i j}-\alpha_{i j}\right) \cdot\left(s_{k l}-\alpha_{k l}\right)-1=0,
$$

where $A$ is a material constant. In this model, the yield locus of a thin-walled tube does not rotate when subjected to either axial extension or pure torsion, in agreement with experimental results. However, in the case of combined loading, even for a proportional loading, the model always predicts a rotation of the subsequent yield surface, which is not substantiated by experimental findings (PHIIIIPS \& TANG [1972]; WU \& YEH [1991]).

LEE and ZAVERL [1978, 1979] used the form

$$
3 F=M_{i j}\left(\sigma_{i}-\alpha_{i}\right) \cdot\left(\sigma_{j}-\alpha_{j}\right)-k^{2}=0,
$$


where $M_{i j}$ is the distortion tensor and $\sigma_{i}$ and $\alpha_{i}$ are vectors formed by the components of stress $\sigma_{i j}$ and back stress $\alpha_{i j}$. REEs [1984] used the yield condition

$$
f=C_{i j} \sigma_{i j}+C_{i j k l} \sigma_{i j} \sigma_{k l}+C_{i j k l m n} \sigma_{i j} \sigma_{k l} \sigma_{m n}-1=0
$$

and showed that the components of the sixth rank anisotropy tensor $C_{i j k l m n}$ are physically significant in presenting the distortion. By employing the method of BALTOV and SAWCZUK [1965], IMATANI and INOUE [1987] used the following expression for the anisotropic part:

$$
\begin{aligned}
A_{i j k l}= & P\left[\alpha_{\sigma}\left(\sigma-\alpha_{\sigma}\right)^{2}+\alpha_{\tau}\left(\tau-\alpha_{\tau}\right)^{2}\right] \\
& +Q\left[\alpha_{\sigma}\left(\sigma-\alpha_{\sigma}\right)+\alpha_{\tau}\left(\tau-\alpha_{\tau}\right)\right]\left[\sigma\left(\sigma-\alpha_{\sigma}\right)+\tau\left(\tau-\alpha_{\tau}\right)\right]-1=0,
\end{aligned}
$$

where $P$ contributed to the description of the Bauschinger effect and $Q$ described the cross effect. In eqn $(5),(\sigma, \tau)$ and $\left(\alpha_{\sigma}, \alpha_{\tau}\right)$ are, respectively, the stress and the back stress components during axial-torsion of a thin-walled cylinder.

In the geometric approach, KURTYKA and ZYCZKowSKI [1985] and KURTYKA [1988] used the five dimensional space of ILYUSHIN [1954] and LENSKY [1960] to describe the subsequent yield surfaces. Phillips and Weng [1975] and Eisenberg and Yen [1984] divided the yield surface into the forward and rear parts and introduced a mapping technique that maps the initial yield surface into subsequent yield surfaces. This mapping was employed by WU and YEH [1987] where they described the distortion of yield surface by use of the endochronic theory.

In this work, it is realized that the incorporation of all factors that lead to anisotropy tend to result in complicated equations that are undesirable in application. Therefore, our effort is to keep the equations simple but realistic as compared to experimental findings. This goal may be achieved by a proper simplification of the distortional behavior of the yield surface.

Experimental results of subsequent yield surfaces (PhILlIPS \& TANG [1972]; PhILlIPS et al. [1974]; WU \& YEH [1991]) show that the forward part of the yield surface inflates and the rear part deflates as the loading proceeds. The main feature of distortion is the decrease in the aspect ratio, which is the sum of rear and forward parts over width. A secondary feature is that the subsequent yield surfaces develop sharp fronts and blunt rears. The extent of sharpness of the front part depends on the material being tested and also on the definition of yield surface. PHILlIPs and TANG [1972] excluded prestress points from the yield surfaces and resulted in less sharp yield surfaces, whereas WU and YEH [1991] included those points and the front part of their yield surfaces looked sharp. It is our opinion that a correct description of the aspect ratio of the yield surface would account for the major part of the deformation induced anisotropy of material. The description of a sharp front and blunt rear is of only secondary importance. By neglecting this secondary factor, the prediction of the model would not be much affected, but at the same time the model would gain the advantage of simplicity. The assumption seems reasonable in this case because the normality of the plastic strain increment of this model is with respect to the initial yield surface and not to the subsequent yield surfaces.

In this article, a modified form of endochronic theory is presented to describe subsequent yield surfaces of initially isotropic metallic materials. Assuming a Mises initial 
yield surface, the subsequent yield surface can expand due to isotropic hardening, translate due to kinematic hardening, and change its aspect ratio due to distortion. In this way, all major observed behaviors of metallic materials can be described. The theoretical results are then compared with the experimental results for three materials.

\section{AN ANISOTROPIC FORM OF ENDOCHRONIC THEORY}

Assuming that materials being considered are plastically incompressible, an anisotropic form of the endochronic constitutive equation derived by WU and YEH [1987] is

$$
s_{i j}=S_{y} \frac{d \varepsilon_{i j}^{p}}{d z}+\int_{0}^{z} \lambda_{i j k l}\left(z-z^{\prime}\right) \frac{d \varepsilon_{k l}^{p}}{d z^{\prime}} d z^{\prime}
$$

where

$$
[\lambda]=\left[\begin{array}{llllll}
\mu & 0 & 0 & 0 & 0 & 0 \\
0 & \mu & 0 & 0 & 0 & 0 \\
0 & 0 & \mu & 0 & 0 & 0 \\
0 & 0 & 0 & \nu & 0 & 0 \\
0 & 0 & 0 & 0 & \nu & 0 \\
0 & 0 & 0 & 0 & 0 & \nu
\end{array}\right],
$$

with

$$
\begin{aligned}
& \mu(z)=\sum_{i} \mu_{i} \exp \left(-m_{i} z\right), \\
& \nu(z)=\sum_{i} \nu_{i} \exp \left(-n_{i} z\right)
\end{aligned}
$$

and

$$
d \varepsilon_{i j}^{p}=d \varepsilon_{i j}-\frac{s_{i j}}{2 G}
$$

In the above equations, $\varepsilon_{i j}$ is strain; $G$ is shear modulus; and $S_{y}$ is a material constant proportional to the initial yield stress in shear. The kernel functions $\mu(z)$ and $\nu(z)$ consist of $n$ exponential terms in which $\mu_{i}, m_{i}, \nu_{i}$, and $n_{i}$ are material constants; $z$ is intrinsic time that is used to register deformation histories, and $\lambda_{i j k l}$ is a fourth order tensor. Note that, when $\mu_{i}=\nu_{i}$ and $m_{i}=n_{i}$, the two kernel functions are equal, that is, $\mu(z)=$ $\nu(z)$, and the following constitutive equation for isotropic rate of kinematic hardening is recovered:

$$
s_{i j}=S_{y} \frac{d \varepsilon_{i j}^{p}}{d z}+\int_{0}^{z} \mu\left(z-z^{\prime}\right) \frac{d \varepsilon_{i j}^{p}}{d z^{\prime}} d z^{\prime}
$$

Equation (6) may be rewritten as 


$$
s_{i j}-\alpha_{i j}=S_{y} \frac{d \varepsilon_{i j}^{p}}{d z}=S_{y} f(z) \frac{d \varepsilon_{i j}^{p}}{d \zeta},
$$

where

$$
\begin{aligned}
f(z) & =\frac{d \zeta}{d z}, \\
d \zeta^{2} & =d \varepsilon^{p} \cdot d \varepsilon^{p},
\end{aligned}
$$

and

$$
\alpha_{i j}=\int_{0}^{z} \lambda_{i j k l}\left(z-z^{\prime}\right) \frac{d \varepsilon_{k l}^{p}}{d z^{\prime}} d z^{\prime} .
$$

The intrinsic time $z$ is scaled by a function $f(z)$, so that another intrinsic time $\zeta$ is defined through eqn (13). The intrinsic time $\zeta$ is further defined in terms of the plastic strain by eqn (14). The function $f(z)$ has been shown to represent strain hardening and is referred to as the hardening function. The back stress $\alpha_{i j}$, given by the integral of eqn (15), specifies the position of the center of yield surface.

From eqn (12), the yield surface is usually represented by

$$
\left(s_{i j}-\alpha_{i j}\right) \cdot\left(s_{i j}-\alpha_{i j}\right)=S_{y}^{2} f(z)^{2} .
$$

If $f(z)$ is isotropic as in VALANIS [1980], then $f(z)$ represents isotropic hardening and $\alpha_{i j}$ the kinematic hardening. Eqn (16) thus describes a combined isotropic-kinematic hardening by use of the Mises yield criterion. On the other hand, the expression:

$$
d \mathbf{e}^{p}=\frac{1}{s_{y}}(\mathbf{s}-\boldsymbol{\alpha}) d z
$$

may be obtained from eqn (12), which is the plastic strain increment and may be regarded as the flow rule.

In the axial-torsional strain space, eqn (14) is reduced to

$$
d \zeta^{2}=k_{1}\left(d \varepsilon^{p}\right)^{2}+k_{2}\left(d \eta^{p}\right)^{2},
$$

where $\varepsilon^{p}$ is the plastic axial strain and $\eta^{p}$ is the plastic tensorial shear strain, $k_{1}=\frac{3}{2}$ and $k_{2}=2$ for initially isotropic materials subject to constant plastic strain-rate deformation.

\section{A MODIFIED THEORY ACCOUNTED FOR YIELD SURFACE DISTORTION}

The endochronic theory presented in the previous section is now modified by redefining the intrinsic time as

$$
d \zeta^{2}=d \boldsymbol{\varepsilon}^{p} \cdot \underline{\mathbf{K}} \cdot d \boldsymbol{\varepsilon}^{p}
$$


instead of the definition given in eqn (14). This definition of intrinsic time is similar to the original one proposed by VALANIS [1971], except that the plastic strain is used instead of the total strain. In eqn (19), $\mathbf{K}$ is a fourth rank material tensor and is positive definite. In all previous investigations, $\mathbf{K}$ was assumed to be isotropic throughout the course of deformation for mathematical simplicity. This assumption is now relaxed so that $\mathbf{K}$ depends on the direction of loading. Eqn (19) simply asserts that the rate of accumulation of the intrinsic time $\zeta$ is not the same, when the material is subjected to shear and when subjected to tension. However, $\mathbf{K}$ is initially isotropic for initially isotropic materials.

Due to the symmetric property of the plastic strain tensor $d \varepsilon^{p}$, tensor $\underline{\mathbf{K}}$ enjoys the following symmetry:

$$
d \varepsilon_{i j}^{p} K_{i j k l} d \varepsilon_{k l}^{p}=d \varepsilon_{k l}^{p} K_{k l i j} d \varepsilon_{i j}^{p}
$$

and

$$
d \varepsilon_{i j}^{p} K_{i j k l} d \varepsilon_{k l}^{p}=d \varepsilon_{i j}^{p} K_{i j l k} d \varepsilon_{l k}^{p}
$$

Thus,

$$
K_{i j k l}=K_{k l i j}=K_{i j l k}
$$

and the number of independent components of the anisotropic tensor $\underset{\mathbf{K}}{\sim}$ is reduced from 81 to 21 .

By the substitution of eqn (17) into (19), the following expression is obtained

$$
\left[(\mathbf{s}-\boldsymbol{\alpha}) \cdot \underline{\mathbf{K}} \cdot(\mathbf{s}-\boldsymbol{\alpha})-S_{y}^{2} f(z)^{2}\right] d \zeta^{2}=0,
$$

which then reduces to

$$
(\mathbf{s}-\boldsymbol{\alpha}) \cdot \underline{\mathbf{K}} \cdot(\mathbf{s}-\boldsymbol{\alpha})=S_{y}^{2} f(z)^{2} \quad \text { with } d \zeta^{2} \neq 0
$$

or

$$
d \zeta^{2}=0
$$

Equation (22a) is the yield criterion of this anisotropic version of the endochronic theory, and eqn (22b) describes the elastic behavior, which may be seen from (10) and (19) due to the positive definiteness of $\underset{\sim}{\mathbf{K}}$. In this version, $\boldsymbol{\alpha}$ still represents the back stress and $f(z)$ the isotropic hardening function. The von Mises yield criterion, eqn (16), is recovered when $\underline{\mathbf{K}}$ is isotropic and given by

$$
K_{i j k l}=K_{1}^{\prime} \delta_{i j} \delta_{k l}+K_{2}^{\prime}\left(\delta_{i k} \delta_{j l}+\delta_{i l} \delta_{j k}\right),
$$

where $K_{1}^{\prime}$ and $K_{2}^{\prime}$ are constants and $\delta_{i j}$ is the Kronecker delta.

Equation (22a) represents a distortion from the Mises yield criterion due to the presence of $\mathbf{K}$. This criterion may be used for the initial as well as the subsequent yielding. In particular, eqn (22a) can accommodate the idea that the initial yield surface is of the type of von Mises, but the subsequent yield surfaces are obtained by the compression and stretching of the Mises yield surface. It should also be pointed out that while the 
plastic strain increment is still given by eqn (17) in this modified theory, this tensor is no longer normal to the yield surface when $\mathbf{K}$ is not isotropic.

An important point to make here is that the definition of intrinsic time directly affects the form of the yield function, so that the intrinsic time defined by (19) leads to a yield function defined by (22a). On the other hand, a slightly different definition of intrinsic time, such as

$$
d \zeta^{2}=d \boldsymbol{\varepsilon}^{p} \cdot \underline{\mathbf{K}} \cdot d \boldsymbol{\varepsilon}^{p}+\operatorname{tr}\left(\mathbf{L} \cdot d \boldsymbol{\varepsilon}^{p}\right) d \zeta
$$

would lead to a yield function given by

$$
(\mathbf{s}-\boldsymbol{\alpha}) \cdot \underset{\sim}{\mathbf{K}} \cdot(\mathbf{s}-\boldsymbol{\alpha})+S_{y} f(z) \operatorname{tr}\left[\mathbf{L} \cdot(\mathbf{s}-\boldsymbol{\alpha}]=S_{y}^{2} f(z)^{2},\right.
$$

where $\mathbb{L}$ is a material tensor.

\section{THE CASE OF AXIAL-TORSIONAL LOADING}

Explicit equations are obtained from the modified theory in the case of axial-torsional loading. This may be accomplished by considering the application of axial-torsional loads to a thin-walled tubular specimen. Because the material is assumed to be plastically incompressible and the cylindrical coordinates $(r, \theta, z)$ are used in this discussion, the conditions $d \varepsilon_{z z}^{p}+d \varepsilon_{r r}^{p}+d \varepsilon_{\theta \theta}^{p}=0$ and $d \varepsilon_{r r}^{p}=d \varepsilon_{\theta \theta}^{p}$ apply. The matrices of the deviatoric stress $\mathbf{s}$, the back stress $\boldsymbol{\alpha}$, and the plastic strain increment $d \boldsymbol{\varepsilon}^{p}$ are

$$
\mathbf{s}=\left[\begin{array}{ccc}
\frac{-\sigma}{3} & 0 & 0 \\
0 & \frac{-\sigma}{3} & \tau \\
0 & \tau & \frac{2 \sigma}{3}
\end{array}\right] \quad \alpha=\left[\begin{array}{ccc}
\frac{-\alpha_{z z}}{2} & 0 & 0 \\
0 & \frac{-\alpha_{z z}}{2} & \alpha_{\theta z} \\
0 & \alpha_{\theta z} & \alpha_{z z}
\end{array}\right]
$$

and

$$
d \varepsilon^{p}=\left[\begin{array}{ccc}
\frac{-d \varepsilon^{p}}{2} & 0 & 0 \\
0 & \frac{-d \varepsilon^{p}}{2} & d \eta^{p} \\
0 & d \eta^{p} & d \varepsilon^{p}
\end{array}\right]
$$

in the cylindrical system. Note that $d \varepsilon_{z z}^{p}=d \varepsilon^{p}$ and the stress tensor $\sigma$ is $(\sigma, \tau)$. The matrices in eqn (26) may also be written as column vectors as:

$$
\begin{aligned}
& {[s]^{T}=\left[\begin{array}{lll}
\frac{-\sigma}{3} & \frac{2 \sigma}{3} & \tau
\end{array}\right], \quad[\alpha]^{T}=\left[\begin{array}{lll}
\frac{-\alpha_{z z}}{2} & \alpha_{z z} & \alpha_{\theta z}
\end{array}\right]} \\
& {[s-\alpha]^{T}=\left[\frac{-1}{2}\left(\frac{2 \sigma}{3}-\alpha_{z z}\right) \quad\left(\frac{2 \sigma}{3}-\alpha_{z z}\right) \quad\left(\tau-\alpha_{\theta z}\right)\right]}
\end{aligned}
$$


and

$$
\left[d \varepsilon^{p}\right]^{T}=\left[\begin{array}{lll}
\frac{-d \varepsilon^{p}}{2} & d \varepsilon^{p} & d \eta^{p}
\end{array}\right]
$$

For the present case, and by observing the symmetry of eqn (20), the tensor $\underset{\sim}{\mathbf{K}}$ can be represented by a square symmetric matrix given by

$$
[K]=\left[\begin{array}{lll}
K_{11} & K_{12} & K_{13} \\
K_{12} & K_{22} & K_{23} \\
K_{13} & K_{23} & K_{33}
\end{array}\right] .
$$

In considering the theory of the form of eqns (19) and (22a), it is now easy to show, by the substitution of (29) and (26) into (19), that

$$
\begin{aligned}
d \zeta^{2} & =d \boldsymbol{\varepsilon}^{p} \cdot \underline{\mathbf{K}} \cdot d \boldsymbol{\varepsilon}^{p} \\
& =\left(\frac{K_{11}}{4}-K_{12}+K_{22}\right)\left(d \varepsilon^{p}\right)^{2}+\left(2 K_{23}-K_{13}\right)\left(d \varepsilon^{p}\right)\left(d \eta^{p}\right)+K_{33}\left(d \eta^{p}\right)^{2} \\
& =K_{1}\left(d \varepsilon^{p}\right)^{2}+2 K_{3}\left(d \varepsilon^{p}\right)\left(d \eta^{p}\right)+K_{2}\left(d \eta^{p}\right)^{2}
\end{aligned}
$$

where

$$
\begin{aligned}
& K_{1}=\frac{K_{11}}{4}-K_{12}+K_{22} \\
& K_{2}=K_{33} \\
& K_{3}=\frac{1}{2}\left(2 K_{23}-K_{13}\right)
\end{aligned}
$$

and that eqn (22a) reduces to

$$
\frac{4}{9} K_{1}\left(\sigma-\frac{3}{2} \alpha_{z z}\right)^{2}+\frac{4}{3} K_{3}\left(\sigma-\frac{3}{2} \alpha_{z z}\right)\left(\tau-\alpha_{\theta z}\right)+K_{2}\left(\tau-\alpha_{\theta z}\right)^{2}=s_{y}^{2} f(z)^{2} .
$$

It is seen that the yield function is an ellipse in the $\sigma$ versus $\tau$ space. As plastic deformation occurs, the ellipse will change its shape, depending on the values of $K_{1}$ and $K_{2}$, when the semimajor and semiminor axes change their lengths. At the same time, isotropic hardening takes place according to the function $f(z)$ and the ellipse translates according to the magnitude of the back stress $\alpha$. In addition, rotation of the yield surface occurs according to the value of $2 K_{3}$. Therefore, the major features of subsequent yield surfaces as observed experimentally by PHILLIPS and TANG [1972] and WU and YEH [1991] can all be described.

Experimental results of Phillips and his coworkers suggested the lack of rotation of the yield surface if preloading was purely axial or purely torsional. No rotation was observed when the purely axial preloading was followed by a shear loading while keeping the axial load constant. This was also true in the corresponding case of pure shear followed by an axial loading while keeping the shear stress constant. Rotation of yield 
surface was observed only when the path of preloading was inclined to the axial and torsional axes in the stress space. Based on the aforementioned observations, it is reasonable to assume that $K_{3}$ is non-zero only when the loading path is inclined. The value of $K_{3}$ must also be zero at initial yielding even for an inclined path.

For initially isotropic materials at initial yielding, eqn (29) and (31) are given by

$$
d \zeta^{2}=K_{1}\left(d \varepsilon^{p}\right)^{2}+K_{2}\left(d \eta^{p}\right)^{2}
$$

and

$$
\frac{4}{9} K_{1} \sigma^{2}+K_{2} \tau^{2}=S_{y}^{2}
$$

where $K_{3}=0$. When $K_{1}=\frac{3}{2}$ and $K_{2}=2$, eqn (32) reduces to eqn (18) and eqn (33) becomes

$$
\sigma^{2}+3 \tau^{2}=\frac{3}{2} S_{y}^{2}
$$

which is the Mises yield function. Therefore, the functions $K_{1}, K_{2}$, and $K_{3}$ have the initial values of $\frac{3}{2}, 2$, and 0 , respectively.

The details of modeling the compression and stretching of yield surfaces are now discussed based on the yield function given by (31). An affine transformation is used either to deform an ellipse into another ellipse with different aspect ratio or rotate an ellipse into one with a different orientation. The equation for an ellipse in the $x_{i}$-frame is

$$
a_{1} x_{1}^{2}+2 a_{3} x_{1} x_{2}+a_{2} x_{2}^{2}=a_{4},
$$

where $a_{i}$ are coefficients defining the shape and size of an ellipse. By scaling the $x_{i}$ with the following relations:

$$
y_{1}=x_{1} / m \quad y_{2}=x_{2}
$$

eqn (3.5) is rewritten as

$$
c_{1} y_{1}^{2}+2 c_{3} y_{1} y_{2}+c_{2} y_{2}^{2}=c_{4} \text {, }
$$

where

$$
c_{1}=a_{1} m^{2} \quad c_{2}=a_{2} \quad c_{3}=a_{3} m \quad c_{4}=a_{4} .
$$

Eqn (37) is an ellipse that has been stretched or compressed and, therefore, its aspect ratio is different than that of (35).

On the other hand, a rotation of the ellipse of (35) through an angle $\phi$ about the $x_{3}$-axis would result in

$$
b_{1} y_{1}^{2}+2 b_{3} y_{1} y_{2}+b_{2} y_{2}^{2}=b_{4}
$$

where

$$
\begin{aligned}
& y_{1}=x_{1} \cos \phi+x_{2} \sin \phi \\
& y_{2}=-x_{1} \sin \phi+x_{2} \cos \phi
\end{aligned}
$$


and

$$
\begin{aligned}
b_{1} & =a_{1} \cos ^{2} \phi+a_{2} \sin ^{2} \phi+2 a_{3} \sin \phi \cos \phi \\
b_{2} & =a_{1} \sin ^{2} \phi+a_{2} \cos ^{2} \phi-2 a_{3} \sin \phi \cos \phi \\
2 b_{3} & =2\left(-a_{1}+a_{2}\right) \sin \phi \cos \phi+2 a_{3}\left(\cos ^{2} \phi-\sin ^{2} \phi\right)
\end{aligned}
$$

The new axes $y_{i}$ are lying in the directions of the major and minor axes of the ellipse.

Each of the above affine transformations is applied to a corresponding reference ellipse. The initial yield surface is the initial reference ellipse, which will remain so until a change of direction in the stress path takes place. Eqn (37) is used to describe the compression or stretching of the ellipse when moving along a straight path. The last ellipse immediately before the change in direction of the stress path is then taken as the reference ellipse for the rotated ellipse governed by eqn (39). Upon the change of direction, transformation (40) applies, and eqn (39) will then describe the rotated yield surface. The compression or stretching of the ellipse along a straight path after the rotation is described by eqn (37), using the ellipse immediately after the rotation and expressed by eqn (39) as the reference ellipse. The actual application of the affine deformation and/or rotation depends entirely on the loading path being investigated. The rotation of axes occurs at each change of direction of the stress path.

To simplify computation, the coordinate frame of the yield surface is assumed to coincide with the frame of the back stress. The distortion of yield surface can be conveniently visualized when there is no isotropic hardening, that is, when $f(z)=1$. The following expression of $m$ is proposed to describe the reduction of the sum of the forward and rear parts of the yield surface during a loading process. As the loading continues, this sum will eventually become steady. The expression is

$$
m=d_{1}-\left(d_{1}-1\right) \exp \left(-d_{2}\left|\alpha_{d}\right|^{2}\right)
$$

where $d_{1}$ and $d_{2}$ are material constants and $\alpha_{d}$ is the difference of the current back stress and the back stress of the corresponding reference ellipse. When the loading path changes its direction, the coordinate frame will rotate through an angle $\phi=\tan ^{-1}\left(d \alpha_{\theta z} / d \alpha_{z z}\right)$. With the help of this angle and eqn (42), the stretching and rotation of the subsequent yield surface may be simulated. In this work, the location of the center of yield surface is determined from experiment throughout the stress path. Therefore, $d \alpha_{\theta z}$ and $d \alpha_{z z}$ are known.

\section{COMPARISON WITH EXPERIMENTAL RESULTS}

In this section, the theory is compared with three sets of experimental data, each for a different material. Each experimental data set involves several preloading paths. By use of the experimental results, which include the stress, the back stress, and the plastic strain, the material constants mentioned in the previous section are determined. It is shown that the theory of this article expressed by eqns (19) and (22) is capable of simulating the data set considered to a reasonable degree of accuracy. The figures of this section are drawn using $\sigma$ as the horizontal axis and $\tau^{\prime}=\sqrt{3} \tau$ as the vertical axis.

Figs. 1-4 show the theoretical results compared with the experimental data of PHILLIPS et al. [1972, 1974]. Eqn (29) is used which, when compared with (33), gives $a_{1}=\frac{2}{3}$, 


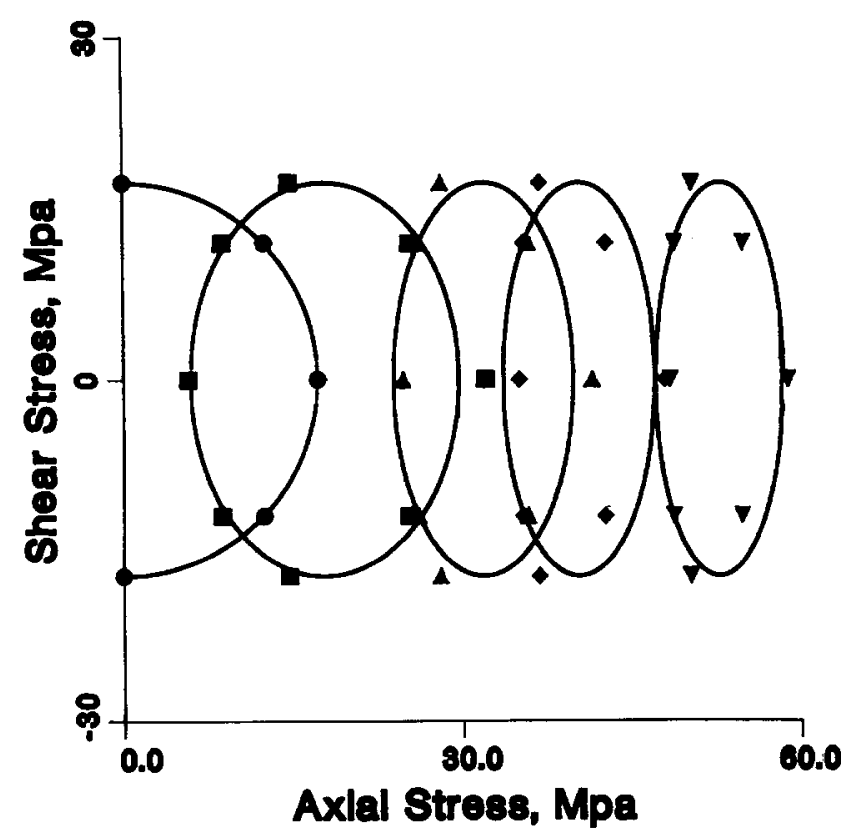

Fig. 1. Yield surfaces with prestress in tension for 1100-0 Al.

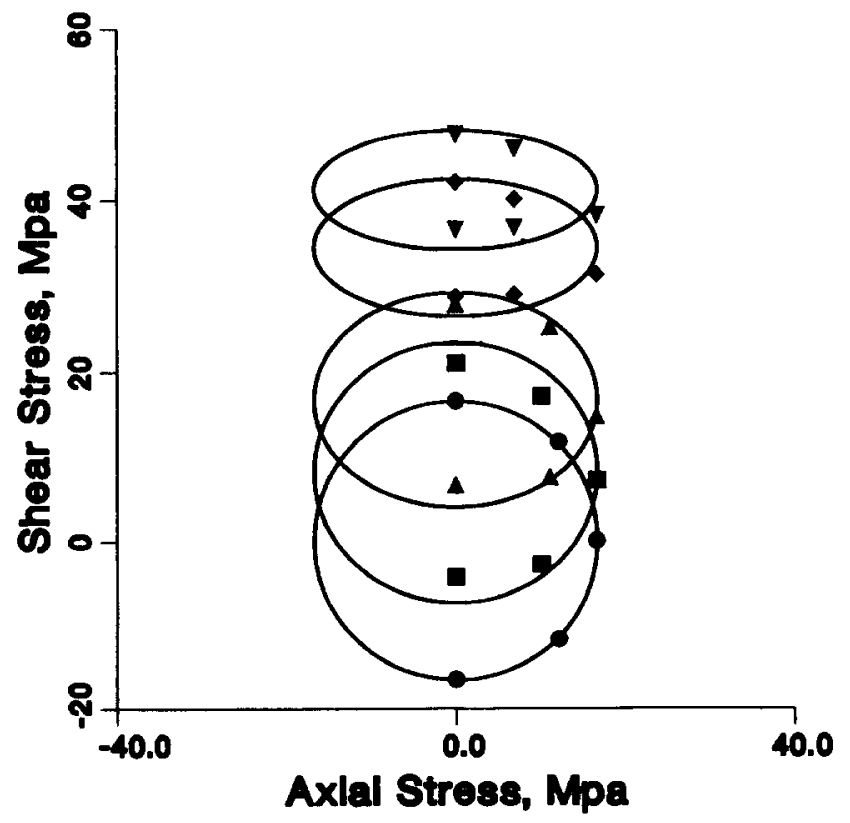

Fig. 2. Yield surfaces with prestress in torsion for 1100-0 Al. 


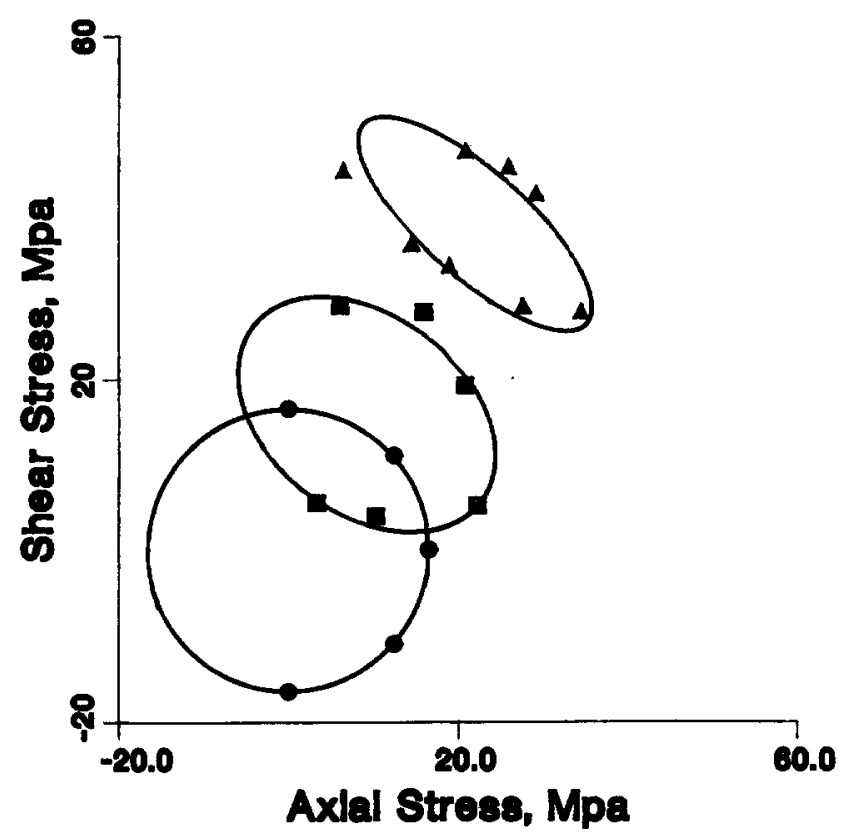

Fig. 3. Yield surfaces with proportional tension-torsion prestress for 1100-0 Al.

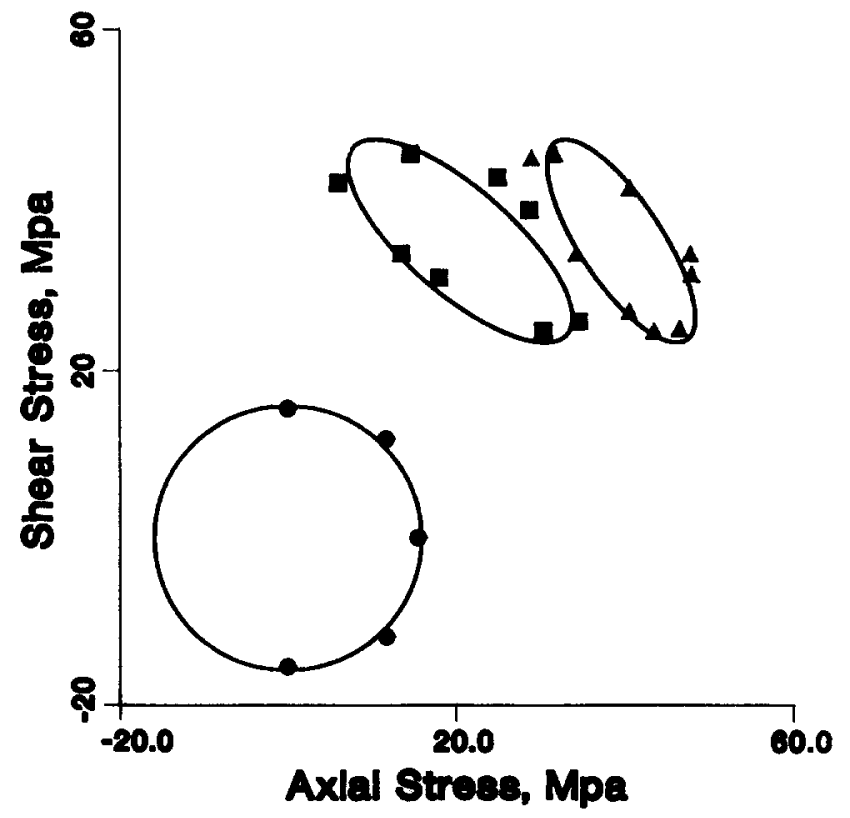

Fig. 4. Proportional tension-torsion prestress followed by tension for 1100-0 Al. 
$a_{2}=2$, and $a_{3}=0$. The material constants of eqn (42) are determined to be $d_{1}=3.4$ and $d_{2}=0.0015(\mathrm{MPa})^{-2}$. These constants are used in the simulation for various paths in this test series. The specimens were made of commercially pure aluminum $1100-0$, and the test data at $70^{\circ} \mathrm{F}$ are of interest here. Figure 1 shows specimen S-5 under monotonic tensile prestress. Four subsequent yield surfaces are simulated (Symbol $\bullet$ denotes 0 prestress, $\square \sigma=37.9, \Delta \sigma=46.5, \diamond \sigma=52.5$, and $\nabla \sigma=63.5 \mathrm{MPa})$. It is seen that the agreement is quite satisfactory. Figure 2 shows the initial and subsequent yield surfaces with prestress in torsion (Symbol $\bullet$ denotes 0 prestrain, $\boldsymbol{\nabla} \tau=12.2, \Delta \tau=16.1, \bullet \tau=$ 24.3 , and $\nabla \tau=27.5 \mathrm{MPa}$ ). The third subsequent yield surface was from specimen S-12, whereas the remaining yield surfaces were from specimen $S-4$. The agreement between the theory and the experiment is again quite good. Figure 3 shows the yield surfaces of specimen S-8 under a combined axial-torsional proportional prestress [Symbol denotes 0 prestress, $\sigma=\tau=18.4 \mathrm{MPa}, \Delta \sigma=\tau=28.2 \mathrm{MPa}$ ), and Fig. 4 shows the results of specimen S-10. This test was subjected to a combined proportional axialtorsional loading followed by an axial preloading (Symbol $\bullet$ denotes 0 prestress, $\square \sigma=$ $\tau=26.9 \mathrm{MPa}, \Delta \sigma=50.8$, and $\tau=19.6 \mathrm{MPa})$. In the analysis, the $x_{1}$-axis was first taken to be in the direction of the proportional stress path and then rotated clockwise through an angle of 60 degrees (in the $\sigma-\tau^{\prime}$ space) at the final stage of prestress. It is seen that the agreement is quite satisfactory for such a complex preloading path.

Experimental data of WU and YEH [1991] for annealed AISI type 304 stainless steel are considered next. In this test series, three specimens were loaded with three different prestress paths. Specimen YA was loaded in pure tension; specimen YS was in pure torsion; and specimen YB was under a combined prestress of proportional tension and torsion. For the description of results of this test series, the material constants are $d_{1}=$ 1.5 and $d_{2}=0.0005(\mathrm{MPa})^{-2}$. Figure 5 shows the initial and the first three subsequent yield surfaces of specimen YA under monotonic tensile prestresses (Symbol $\bullet$ denotes 0 prestrain, $\square d \varepsilon^{p}=540 \times 10^{-6}, \Delta d \varepsilon^{p}=2232 \times 10^{-6}$, and $\left.\bullet d \varepsilon^{p}=4184 \times 10^{-6}\right)$; Fig. 6 shows the initial and the first, the second, the fourth, and the seventh subsequent yield surfaces with prestresses in torsion (Symbol - denotes 0 prestrain, $\square d \eta^{p}=35 \times$ $10^{-6}, d \eta^{p}=85 \times 10^{-6}, \Delta d \eta^{p}=727 \times 10^{-6}$, and $\bullet d \eta^{p}=3809 \times 10^{-6}$ ); and Fig. 7 shows the initial and the first four subsequent yield surfaces of specimen YB under combined tension-torsional prestresses (Symbol $\bullet$ denotes 0 prestrain, $\square d \varepsilon^{p}=47 \times 10^{-6}$ and $d \eta^{p}=64 \times 10^{-6}, \Delta d \varepsilon^{p}=283 \times 10^{-6}$ and $d \eta^{p}=357 \times 10^{-6}, \Delta d \varepsilon^{p}=1502 \times 10^{-6}$ and $d \eta^{p}=2249 \times 10^{-6}, \nabla d \varepsilon^{p}=5241 \times 10^{-6}$ and $\left.d \eta^{p}=7897 \times 10^{-6}\right)$. The agreement between theory and experiment is quite good when the prestress point is not considered a point on the yield surface. As previously mentioned, PHILLIPS and TANG [1972] assumed that the yield surface would not include the prestress point; but WU and YEH [1991] argued that the yield surface should include the prestress point, because in a continuous loading, the load is always at the prestress point. If it is so, then the sharp-front effect of the subsequent yield surface is quite pronounced and is not well described by our model for this material.

Finally, the experimental results of Wu et al. [1992] are simulated. In this test series, specimens were made of annealed isotropic high purity cast aluminum. This material shows significant isotropic hardening which is described by eqn (13). A step-by-step calculation is used. The intrinsic time increment $d z$ is calculated from eqn (17), and $d \zeta$ is from (29). For each step, the value of $m$ is first determined from (42) with $d_{1}=1.43$ and $d_{2}=0.021(\mathrm{MPa})^{-2}$. The $c_{i}$ s are then calculated from (38) in the case when the ellipse is undergoing deformation. In the case of rotation of the ellipse, the angle $\phi$ is 


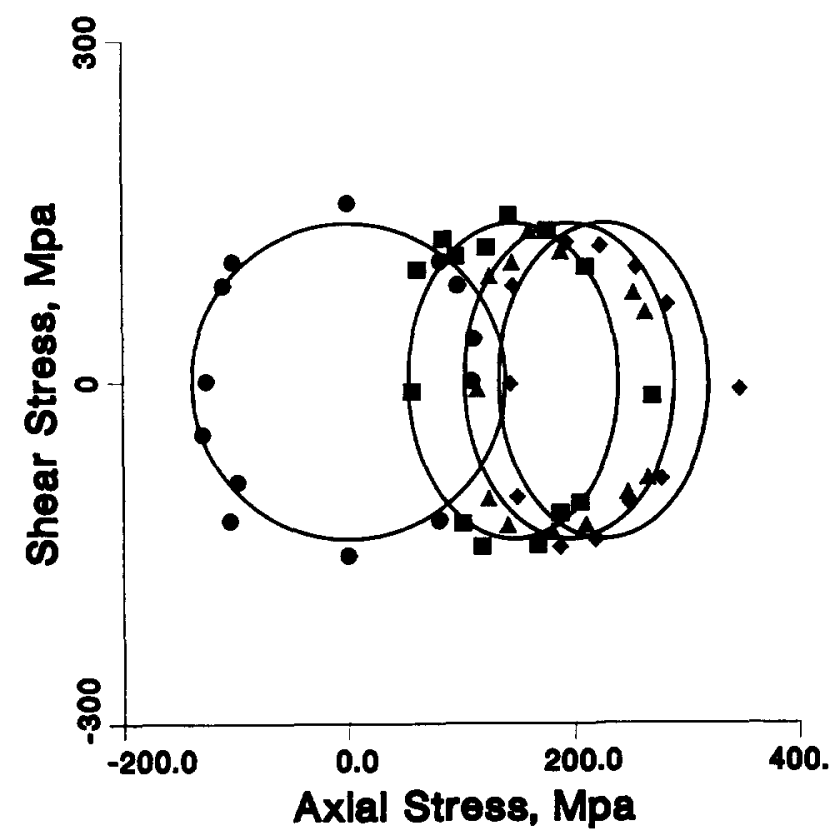

Fig. 5. Yield surfaces with prestress in tension for 304 stainless steel.

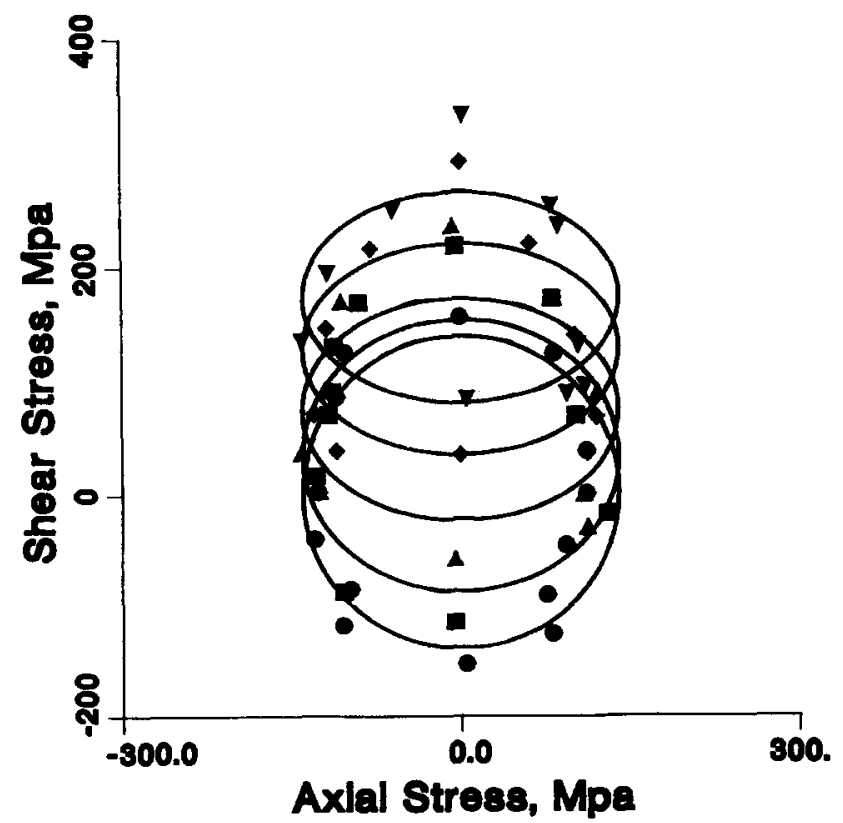

Fig. 6. Yield surfaces with prestress in torsion for 304 stainless steel. 


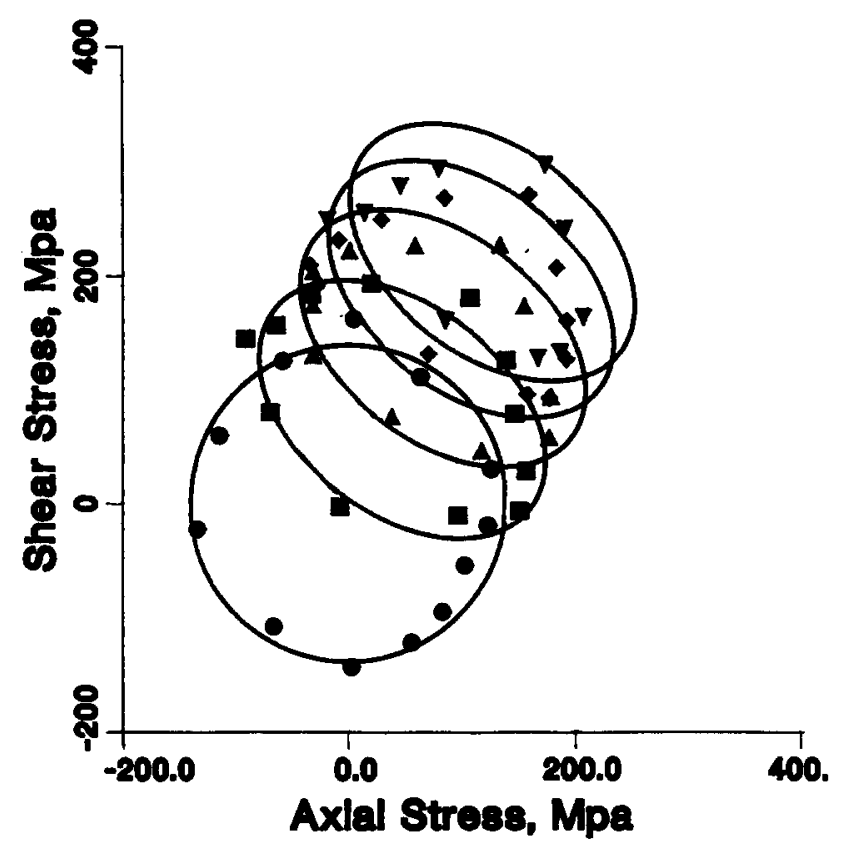

Fig. 7. Yield surfaces with proportional tension-torsion prestress for 304 stainless steel.

first determined from the back stress, eqn (41) is then used to compute the $b_{i} s$. The current values of $K_{i}$ s are then found by comparing (31) with (37) in the case of deformation or with (39) in the case of rotation of the ellipse. Their initial values have been given earlier to be $K_{1}=1.5, K_{2}=2$, and $K_{3}=0$.

Fig. 8 shows the yield surfaces of a specimen with several tensile prestresses (Symbol $\bullet$ denotes 0 prestrain, $\square d \varepsilon^{p}=0.58 \times 10^{-2}, \Delta d \varepsilon^{p}=2.0 \times 10^{-2}$, and $\bullet d \varepsilon^{p}=5.8 \times$ $10^{-2}$ ). In this case, $d \varepsilon^{p} \neq 0$ and $d \eta^{p}=0$, and (17) and (29) reduce, respectively, to

$$
d z=\frac{S_{y} d \varepsilon^{p}}{\left(\frac{2}{3} \sigma-\alpha_{z z}\right)}
$$

and

$$
d \zeta^{2}=K_{1}\left(d \varepsilon^{p}\right)^{2}
$$

Fig. 9 shows that of a specimen with prestresses in torsion with $d \eta^{p} \neq 0$ and $d \varepsilon^{p}=0$ (Symbol $\bullet$ denotes 0 prestrain, $\square d \eta^{p}=0.5 \times 10^{-2}$, and $\Delta d \eta^{p}=5.0 \times 10^{-2}$ ). In this case, eqns (17) and (29) reduce to

$$
d z=\frac{S_{y} d \eta^{p}}{\left(\tau-\alpha_{\theta z}\right)}
$$

and

$$
d \zeta^{2}=K_{2}\left(d \eta^{p}\right)^{2}
$$




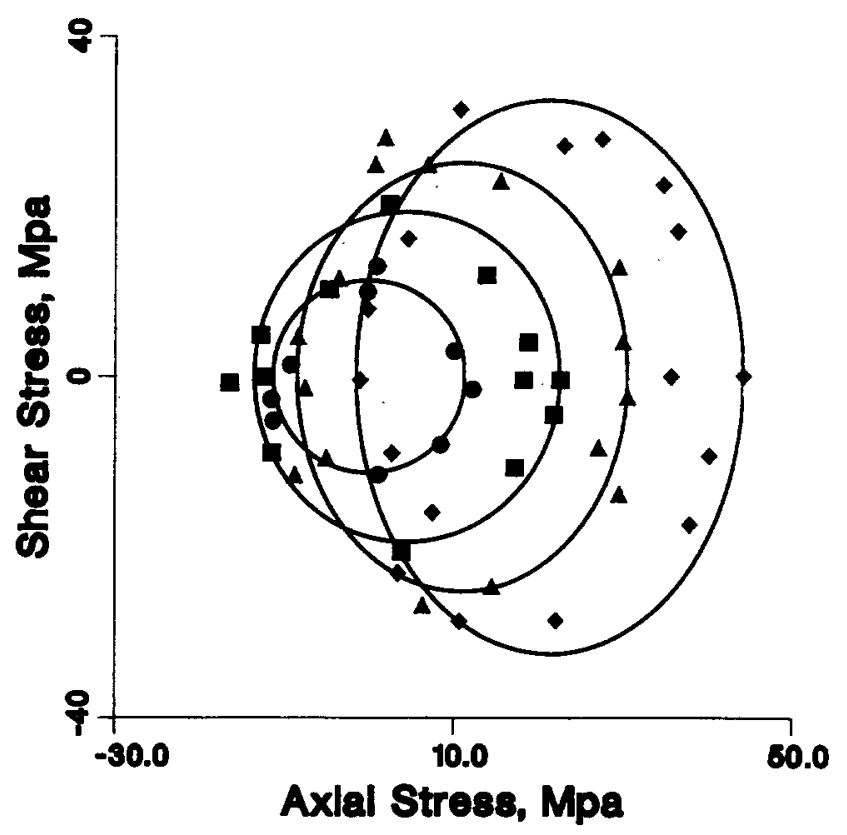

Fig. 8. Yield surfaces with prestress in tension for high purity aluminum.

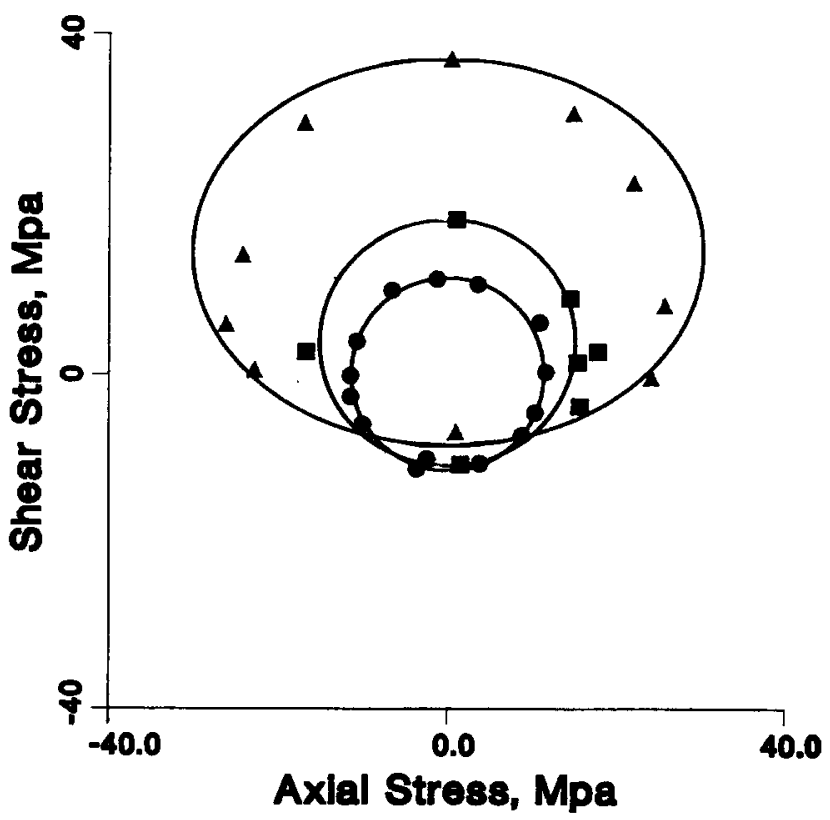

Fig. 9. Yield surfaces with prestress in torsion for high purity aluminum. 
By putting the values of $d z$ and $d \zeta$ from (43-46) into eqn (13), the current value of $f(z)$ can be obtained. Finally, eqn (31) is plotted in Figs. 8 and 9. The agreement between the theoretical and experimental results are quite good for this test series.

\section{CONCLUDING REMARKS}

The version of endochronic theory discussed in this article leads to an anisotropic yield function that is capable of describing the expansion, motion, and distortion of the yield surface. Although the motion includes the translation and rotation, the distortion includes only the deformation (compression or stretching) of the Mises yield surface. The direction of the plastic flow is independent of the shape of the subsequent yield surface and is in the radial direction emanating from the center of the current yield surface. A new definition of intrinsic time is given that is appropriate for the anisotropic yield function. Other definitions may be used. The different expressions for intrinsic time specify the different degrees of anisotropy that the theory is capable of describing. An isotropic definition of intrinsic time, which was used in most of the previous studies, limits the theory to a combined isotropic-kinematic hardening rule.

Due to the nature of this study, stress space has been used. In other applications, with information supplied in the plastic strain space, eqns (12), (13), (19), and the differential form of (15) may be used to find the corresponding stresses.

Acknowledgements - This study was supported by the National Science Council of Taiwan, R.O.C.

\section{REFERENCES}

1954 Ilyushin, A.A., Prikl. Mat. Mekh., 18, 641 (in Russian).

1960 LENSKY, V.S., “Analysis of Plastic Behavior of Metals Under Complex Loading,” In LeE, E.H., and Symonds, P.S. (eds.), Plasticity, Proc. 2nd Symp. Naval Struct. Meth., Brown University, Rhode Island, pp. 259.

1965 Baltov, A., and Sawczuk, A., “A Rule of Anisotropic Hardening," Acta Mech., 1, 81.

1971 Valanis, K.C., "A Theory of Viscoplasticity Without a Yield Surface, Part I: General Theory; Part II: Application to Mechanical Behavior of Metal," Arch. Mech., 23, 517.

1972 Phillips, A., and TANG, J.L., "The Effect of Loading Path on the Yield Surface at Elevated Temperatures," Int. J. Solids Struct. 8, 463.

1974 Phillips, A., Tang, J.L., and Riccruti, M., "Some New Observations on Yield Surface," Acta Mech., 20, 23.

1975 PhilıIPs, A., and Weng, G.J., “An Analysis Study of An Experimentally Verified Hardening Law," J. Appl. Mech., 42, 375.

1978 LeE, D., and ZAVERL, F., Jr., "A Generalized Strain Rate Dependent Constitutive Equation for Anisotropic Metals," Acta Metal., 26, 1771.

1979 LEE, D., and ZaverL, F., Jr., "A Description of History Dependent Plastic Flow Behavior of Anisotropic Metals," J. Eng. Mat. Tech., 101, 59.

1980 Valanis, K.C., "Fundamental Consequences of a New Intrinsic Time Measure Plasticity as a Limit of the Endochronic Theory," Arch. Mech., 32, 171.

1984 Eisenberg, M.A., and Yen, C.F., “The Anisotropic Deformation of Yield Surface," J. Eng. Mat. Tech., 106, 355.

1984 REES, D.W.A., “An Examination of Yield Surface Distortion and Translation," Acta Mech., 52, 15.

1985 KurtykA, T., and Zyczkowskı, M., "A Geometric Description of Distortional Plastic Hardening of Deviatoric Materials," Arch. Mech., 37, 383.

1986 WU, H.C., YAO, J.C., and CHU, S.C., "Investigation of Endochronic Constitutive Equation Subject to Plastic Strain-Controlled Axial-Torsional Deformation,” J. Eng. Mat. Tech., 108, 262.

1987 Imatani, S., and InOue, T., "Material Anisotropy Induced by Prior Plastic Deformation," In DesaI, C.S., et al., (eds.), Constitutive Laws for Engineering Materials: Theory and Applications, Elsevier Science, New York, p. 573. 
1987 WU, H.C., and YEH, W.C., "Some Considerations in the Endochronic Description of Anisotropic Hardening," Acta Mech., 69, 59.

1988 KURTYKA, T., "Parameter Identification of A Distortional Model of Subsequent Yield Surfaces," Arch. Mech., 40, 433.

1989 WU, H.C., YEH, W.C., "Further Verification of Endochronic Theory Under Biaxial Load," J. Eng. Mat. Tech., 111, 115.

1991 WU, H.C., YEH, W.C., "On the Experimental Determination of Yield Surfaces and Some Results of Annealed 304 Stainless Steel," Int. J. Plasticity, 7, 803.

1992 Wu, H.C., XU, Z.Y., and Ho, C.C., "An Experimental Study of Biaxial Stress-Strain Behavior and Subsequent Yield Surfaces for Aluminum in the Large Strain Range," Research Report No. 92-2, submitted to Alcoa Tech Center, Dept. of Civil Engineering, The University of lowa, Iowa City, IA.

Department of Civil Engineering

National Taiwan University

Taipei, Taiwan, R.O.C.

The University of Iowa

Iowa City, IA 52242, USA

(Received in final revised form 21 June 1994) 